\title{
Effect of the nature of randomness on quenching dynamics of Ising model on complex networks
}

\author{
Soham Biswas, 1 田 and Parongama Sen ${ }^{1, \text { 由 }}$ \\ ${ }^{1}$ Department of Physics, University of Calcutta, 92 Acharya Prafulla Chandra Road, Kolkata 700009, India
}

\begin{abstract}
Randomness is known to affect the dynamical behaviour of many systems to a large extent. In this paper we investigate how the nature of randomness affects the dynamics in a zero temperature quench of Ising model on two types of random networks. In both the networks, which are embedded in a one dimensional space, the first neighbour connections exist and the average degree is four per node. In the random model A, the second neighbour connections are rewired with a probability $p$ while in the random model B, additional connections between neighbours at Euclidean distance $l(l>$ 1) are introduced with a probability $P(l) \propto l^{-\alpha}$. We find that for both models, the dynamics leads to freezing such that the system gets locked in a disordered state. The point at which the disorder of the nonequilibrium steady state is maximum is located. Behaviour of dynamical quantities like residual energy, order parameter and persistence are discussed and compared. Overall, the behaviour of physical quantities are similar although subtle differences are observed due to the difference in the nature of randomness.
\end{abstract}

PACS numbers: 89.75.Hc, 75.78.Fg, 81.40.Gh, 64.60.aq, 05.50.+q

\section{INTRODUCTION}

The dynamics of Ising models is a much studied phenomenon and has emerged as a rich field of present day research. An important dynamical feature commonly studied is the quenching phenomenon below the critical temperature. In a quenching process, the system has a disordered initial configuration corresponding to a high temperature and its temperature is suddenly dropped. This results in quite a few interesting phenomena like domain growth [1, 2], persistence [3-6] etc.

In one dimension, a zero temperature quench of the Ising model starting with completely random configuration (which corresponds to a very high temperature) and evolving according to the usual Glauber dynamics, always leads the system to the equilibrium configuration (all spins up or all spins down). The average domain size $D$ increases in time $t$ as $D(t) \sim t^{1 / z}$, where $z$ is the dynamical exponent associated with the growth. As the system coarsens, the magnetisation also grows in time as $m(t) \sim t^{1 / 2 z}$. In two or higher dimensions, however, the system does not always reach equilibrium [6] although these scaling relations still hold good. In zero temperature quench, another important dynamical behavior commonly studied is persistence, which is the probability that a spin has not flipped till time $t$. In regular lattices, in one or higher dimensions, the persistence probability $P(t)$ at time $t$ is usually seen to follow a power law decay given by $P(t) \propto t^{-\theta}$. $\theta$ is called the persistence exponent and is unrelated to any other known static or dynamic exponents.

The dynamical behaviour of Ising models may change drastically when randomness is introduced in the system.

\footnotetext{
*Electronic address: soham.physics@gmail.com
}

${ }^{\dagger}$ Electronic address: psphy@caluniv.ac.in
Randomness can occur in many ways and its effect on dynamics can depend on its precise nature. For example, randomness in the Ising model can be incorporated by introducing dilution in the site or bond occupancy in regular lattices and consequently the percolation transition plays an important role [7, 8]. Scaling behaviour is completely different from power laws here. One can also consider the interactions to be randomly distributed, either all ferromagnetic type or mixed type (e.g., as in a spin glass) [9]; the system goes to a frozen state following a zero temperature quenching in both cases. Another way to introduce randomness is to consider a random field in which case the scaling behaviour is also completely different from power laws [10].

Here we consider Ising models on random graphs or networks where the nearest neighbour connections exist. In addition, the spins have random long range interactions which are quenched in nature. In general, here, the dynamics, instead of leading the system towards its equilibrium state, makes it freeze into a metastable state such that the dynamical quantities attain saturation values different from their equilibrium values.

Moreover, rather than showing a conventional power law decay or growth, the dynamical quantities exhibit completely different behaviour in time.

A point to be noted here is, when long range links are introduced, the domains are no longer well-defined as interacting neighbours could be well separated in space. This results in freezing of Ising spins on random graphs as well as on small world networks [11, 12]. The phase ordering dynamics of the Ising model on a Watts-Strogatz network [13], after a quench to zero temperature, produces dynamically frozen configurations, disordered at large length scales 12, 14]. Even on small world networks, the dynamics can depend on the nature of the randomness; it was observed that while in a sparse network there is freezing, in a densely connected network freezing disappears in the thermodynamic limit [15]. 
In this paper, we investigate the dynamical behaviour of an Ising system on two different networks following a zero temperature quench. In these two networks, both of which are sparsely connected, the nature of randomness is subtly different and we study whether this difference has any effect on the dynamics. Both these networks are embedded in a one dimensional lattice and the nearest neighbour connections always exist and the nodes have degree four on an average. They differ as in one of the networks, the random long range interactions have a spatial dependence. It may be mentioned here that quenching dynamics on such Euclidean networks has not been considered earlier to the best of our knowledge.

It is also quite well known that many dynamical social phenomena can be appropriately mapped to dynamics of spin systems. At the same time, social systems have been shown to behave like complex networks (having small world and/or scale free features etc.). So the present study may be particularly interesting in the context of studying social phenomena described by Ising-type models.

In section II we have described the two different networks which we call random model A (RMA) and random model B (RMB). In Section III we have given a list of the quantities calculated. In section IV and $\mathrm{V}$ we have discussed the detailed dynamical behaviour of Ising spin systems on random model A and random model B respectively. The comparison of the results of the quenching dynamics between the two models are discussed in section VI. In addition, a qualitative analysis of the quenching dynamics is also presented. Summary and concluding statements are made in the last section.

\section{DESCRIPTION OF THE NETWORK MODELS}

The two network models under consideration were introduced in reference [16]. The random model A (RMA) is in fact very similar to the Watts-Strogatz network [13]. Here initially a spin is connected to its four nearest neighbours and then only the second nearest neighbour links are rewired with probability $p$ (Fig. 1). In the RMB, each spin is connected to its two nearest neighbour and then two extra bonds (on an average) are attached randomly to each spin. The extra bonds are attached to spins located at a distance $l>1$ with probability $P(l) \propto l^{-\alpha}$ (Fig. 1). We keep the first neigbours intact in both cases to ensure that the networks are connected. Average degree per node is four in both the networks. The dynamical evolution is considered on the static networks after the process of rewiring/addition of links is completed.

The general form of the Hamiltonian in a one dimensional Ising spin system for RMA and RMB can be written as

$$
H=-\sum_{i<j} J_{i j} S_{i} S_{j}
$$
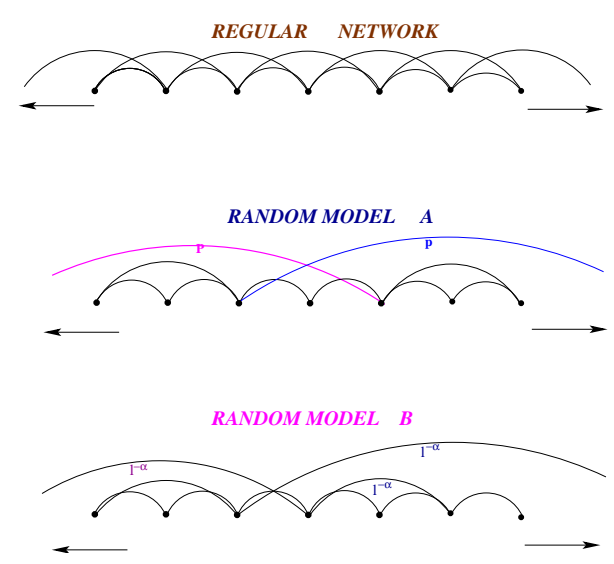

FIG. 1: (Color online) Schematic diagram for different network models. Average degree is $2 \mathrm{~K}=4$ in each network. In the regular network both the first and second nearest neighbours are present. In random model A only second neighbours are rewired with probability p. In random model B first nearest neighbours are always linked while other nodes are linked with the probability $l^{-\alpha}$ with $l \geq 2$.

where $S_{i}= \pm 1$ and $J_{i j}=J$ when sites $i$ and $j$ are connected and zero otherwise. (We take $J=1$ in this paper.) The ground state (minimum energy state at zero temperature) of the Ising spin system in both RMA and RMB is a state with all spins up or all spins down.

RMA is a variant of WS model with identical static properties. It is regular for $p=0$, random for $p=1$, and for any $p>0$, the nature of RMA is small world like [13, 16]. Euclidean models of RMB type have been studied in a few earlier works [16 18]. While it is more or less agreed that for $\alpha \leq 1$, the network is random and for $\alpha>2$, it behaves as a regular network, the nature of the network for intermediate values of $\alpha$ is not very well understood. According to the earlier studies [1618], it may either have a small world characteristic or behave like a finite dimensional lattice. In the present work, we assume that RMB has random nature for $\alpha<1$ and for $1<\alpha<2$, it is small world like (at least for the system sizes considered here) following the results of [16], which is based on exact numerical evaluation of shortest distance and clustering coefficients. This is also because the Euclidean model considered in [16] is exactly identical to RMB with average degree four, while the average degree of the Euclidean models considered in the other earlier studies is not necessarily equal to four.

In case of RMA, the network is regular and random for only two extreme values $p=0$ and $p=1$ respectively, whereas for RMB, the random and regular behaviour of the network are observed over an extended region. The regular network corresponding to these two models is the one dimensional Ising spin system with nearest neighbour and next nearest neighbour interactions. We have studied the zero temperature quenching dynamics for this model also, and the results for the dynamics are identical to that of the nearest neighbour Ising spin model. 
So it will be interesting to note how the dynamics is affected by the introduction of randomness in the Ising spin system and also how the difference in the nature of randomness of the two models RMA and RMB shows up in the dynamics.

In the simulations, single spin flip Glauber dynamics is used in both cases, the spins are oriented randomly in the initial state. We have taken one dimensional lattices of size $L$ with $100 \leq L \leq 1500$ to study the dynamics. The results are averaged over (a) different initial configurations and (b) different network configurations. For each system size the number of networks considered is fifty and for each network the number of initial configuration is also fifty. Periodic boundary condition has been used.

\section{QUANTITIES CALCULATED}

We have estimated the following quantities in the present work.

1. Magnetisation $m(t)$ : For a Ising spin system with regular connections and having only the ferromagnetic interaction, the order parameter is usually the magnetisation, $m=\frac{\left|\sum_{i} S_{i}\right|}{L} . L$ is the size of the system. Magnetisation can be considered as the order parameter, even when the connections are random. We have calculated the growth of magnetisation with time and also the variation of the saturation value of the magnetisation, $m_{\text {sat }}$, with $p$ and $\alpha$ for RMA and RMB respectively.

2. Persistence probability $P(t)$ : As already mentioned, this is the probability that a spin does not flip till time $t$.

3. Energy $E(t)$ : In these networks, domain wall measurement is not very significant, as domains are illdefined. The presence of domain walls in regular lattices causes an energy cost [14]. So instead of the number of domain walls, the appropriate measure for disorder is the residual energy per spin $\varepsilon=E-E_{0}=E+4$, where $E_{0}=-4$ is the known ground state energy per spin and $E$ is the energy of the dynamically evolving state. In fact, the magnetisation is not a good measure of the disorder either, since even when the energy is close to the ground state, magnetisation may be very close to zero (this is also true for the models without randomness). So residual energy measurement is the best way to find out whether the system has reached the equilibrium ground state or it is stuck in a higher energy nonequilibrium steady state. We have measured the decay of residual energy $\varepsilon$ with time and the variation of its saturation value, $\varepsilon_{\text {sat }}$, with $p$ and $\alpha$ for RMA and RMB respectively.

4. Freezing probability: The probability with which any configuration freezes, i.e., does not reach the ground state (the state with magnetisation $m=1$ or the state with zero residual energy) is defined as the freezing probability.

5. Saturation time : It is the time taken by the system to reach the steady state. It has been observed in some earlier studies [19] that it also shows a scaling behaviour with the system size with the dynamical exponent $z$. This in fact provides an alternative method to estimate $z$ when straight forward methods fail.

Both magntisation and energy are regarded as dimensionless quantities ( $\epsilon$ and $E$ scaled by $J$ ) in this paper.

\section{DETAILED RESULTS OF QUENCHING DYNAMICS ON RMA}

The results of a zero temperature quench for the Ising model on the RMA are presented in this section. Starting from a initial random configuration following a quench to zero temperature the system cannot reach the ground state (the state with zero residual energy) always for any $p \neq 0$. The magnetization, energy, persistence all attain a saturation value in time. The saturation values of all the quantities show nonmonotonic behaviour as a function of $p$.

Figure 2 shows the decay of residual energy per spin and the growth of magnetisation with time for different values of the rewiring probability.
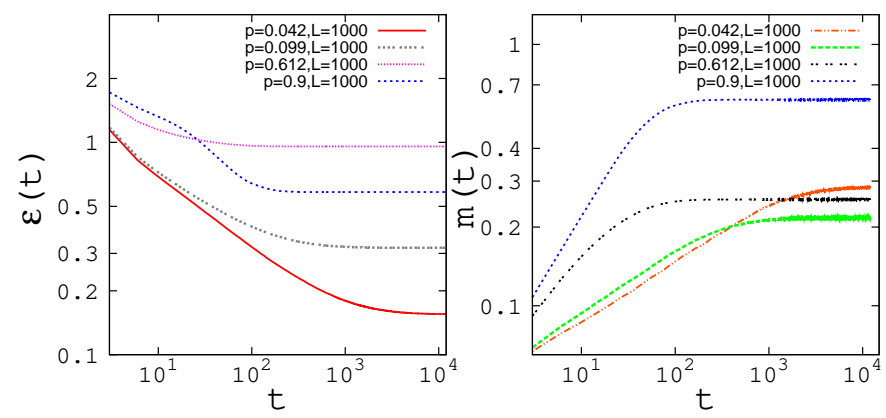

FIG. 2: (Color online) Decay of residual energy per spin and the growth of magnetisation with time for RMA for different probabilities.

It is to be noted that the dynamic quantities do not show any obvious power law behaviour beyond a few time steps. For small $p$, there is apparently a power law behaviour for a larger range of time which we believe is the effect of the $p=0$ point where such a scaling definitely exists. 
The saturation value of the residual energy per spin $\varepsilon_{\text {sat }}$ increases with the rewiring probability $p$ (for small $p$ ), reaches a maximum for an intermediate value of $p$ $(p<1)$ and then decreases again. This implies that the disorder of the spin system is maximum for a non trivial value of $p=p_{\text {maxdis }}$, which can be termed as the point of maximum disorder. The saturation value of magnetization on the other hand decreases for small $p$ and takes its minimum value for another intermediate value of $p(p<1)$, and then increases again (Fig. 33).

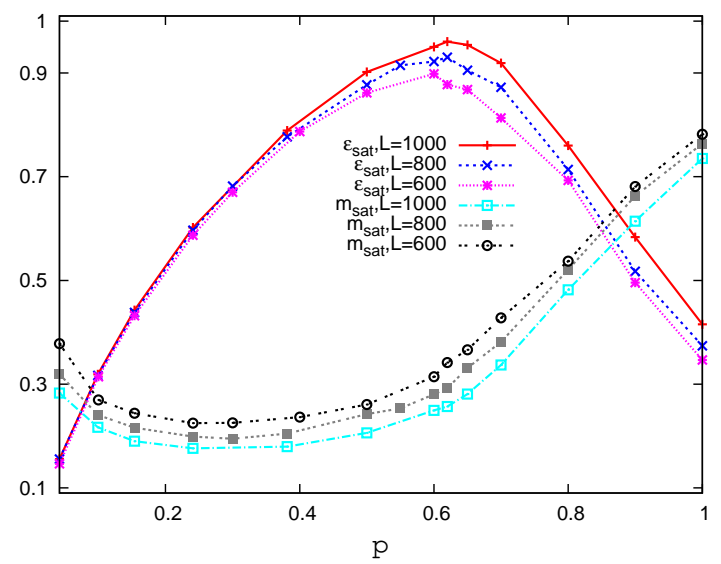

FIG. 3: (Color online) Saturation value of residual energy per spin $\varepsilon_{\text {sat }}$ and the saturation value of magnetisation $m_{\text {sat }}$ is plotted with the probability of rewiring $p$ for Random Model A.

$p_{\text {maxdis }}$ increases with the system size $L$ for small $L$ and then appears to saturate for larger system sizes. The value of the residual energy at $p_{\operatorname{maxdis}}$ also increases with the system size (Fig. 4). This establishes the existence of the point of maximum disorder at an intermediate value of $p(p \simeq 0.62)$ even in the thermodynamic limit.

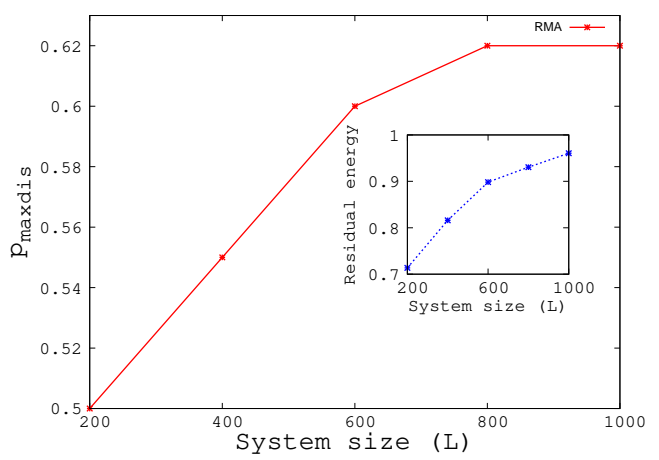

FIG. 4: (Color online) Rewiring probability at the point of maximum disorder is plotted with the system size. Inset shows the increase of residual energy at the point of maximum disorder with the increment of the system size.

Magnetisation reaches a minimum at a value of $p$ which is less than $p_{\text {maxdis }}$. This implies that there exists a region where both magnetisation and energy increase as $p$ increases. This is also apparent from Fig 3 , The phys- ical phenomena responsible for this intriguing feature is conjectured and discussed in detail in sec VI B.

The saturation time decreases very fast with the rewiring probability $p$ for small $p$ and remains almost constant as $p$ increases (Fig. 5). It is known that for $p=0$ the saturation time varies as $L^{2}$, here it appears that for any $p>0$, there is no noticeable size dependence.

For RMA, the freezing probability is almost unity for small $p$. However, when the disorder is increased beyond $p \simeq 0.5$, the freezing probability shows a rapid decrease (Fig: 5, inset). In one dimension, we checked that the freezing probability is zero for the regular network $(p=0)$, but here we find that even for very small values of $p$, the freezing probability is unity. So there is a discontinuty in the freezing probability at $p=0$. This also supports the fact that any finite $p$ can make the dynamics different from a conventional coarsening process.

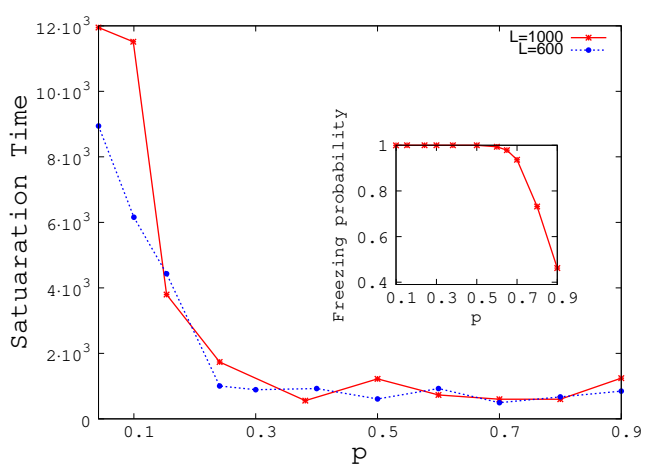

FIG. 5: (Color online) Time of saturation with the probability of rewiring is plotted for two different sizes for Random Model A. Inset shows the variation of freezing probability with the probability of rewiring for RMA.

An interesting observation may be made about the behaviour of the saturation value of the residual energy in the region $p<0.5$. If one allows $p$ to decrease from 0.5 to 0 , the saturation value of the residual energy also decreases although the freezing probability is unity in the entire region. This implies that in this range of the parameter, although the system does not reach the real ground state in any realisation of the network (or initial configuration), such that $\epsilon \neq 0$ in each case, the system has a tendency to approach the the actual ground state monotonically with $p$ for $p<0.5$ (Fig. 3).

The persistence probability follows a stretched exponential behaviour with time for any non zero $p$, fitting quite well to the form

$$
P(t)-P_{\text {sat }} \simeq a \exp \left(-b t^{c}\right) .
$$

The saturation value of the persistence is $P_{\text {sat }}$, and it does not depend on the system size. $P_{\text {sat }}$ changes with the rewiring probability $p$ and there also exists an intermediate value of $p$ where the value of $P_{\text {sat }}$ is maximum. $b$ and $c$ vary nonmonotonically with $p$ (Fig. 6). 


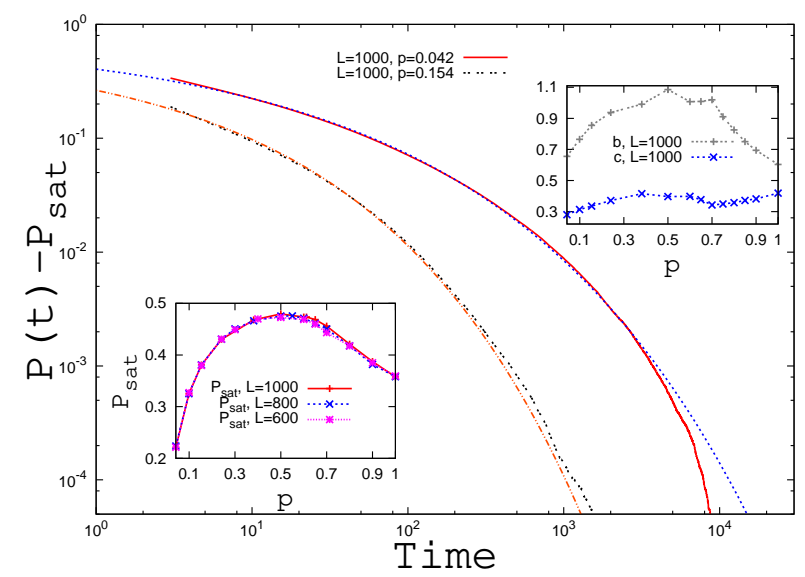

FIG. 6: (Color online) Decay of $P(t)-P_{\text {sat }}$ with time $t$ alongwith the stretched exponential function found to fit its form are shown. The inset in the bottom left shows the variation of the saturation value of persistence $P_{\text {sat }}$ with $p$. The other inset on the top right shows the variation of $b$ and $c$ with $p$.

\section{DETAILED RESULTS OF QUENCHING DYNAMICS ON RMB}

In this section we will present the results of the zero temperature quenching dynamics of Ising model on RMB. Here also the system does not reach the ground state always for any finite value of $\alpha$. The magnetization, energy, persistence all attain a saturation value in time as in RMA. Figure 7 shows the decay of residual energy per spin and the growth of magnetisation with time for different values of $\alpha$. It is to be noted that the dynamical quantities do not show any obvious power law behaviour also for RMB.
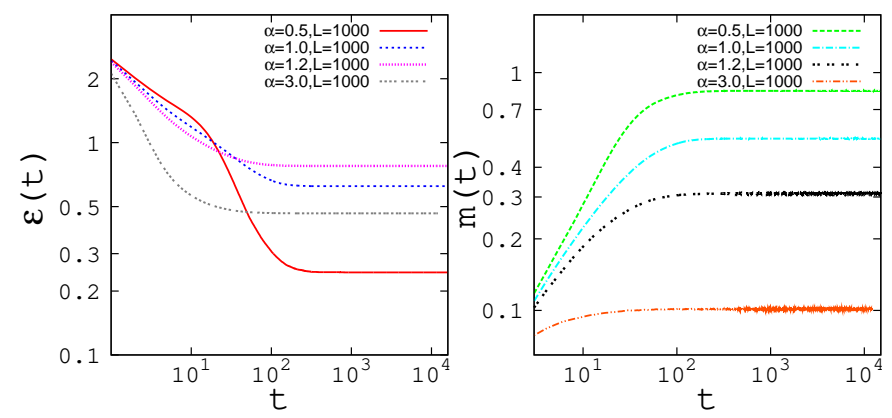

FIG. 7: (Color online) Decay of residual energy per spin and the growth of magnetisation with time for RMB for different probabilities.

The saturation values of all the quantities show nonmonotonic behaviour as a function of $\alpha$. The saturation value of residual energy per spin $\varepsilon_{\text {sat }}$ increases with $\alpha$ for small $\alpha$, reaches a maximum for a finite value of $\alpha$ and then decreases again. This implies that for the RMB also, the disorder of the spin system is maximum for a finite value of $\alpha$, which is the point of maximum disorder here. On the other hand, the saturation value of the magnetization decreases for small $\alpha$ and takes its minimum value for another finite value of $\alpha$ and then slowly increases (Fig. 8).

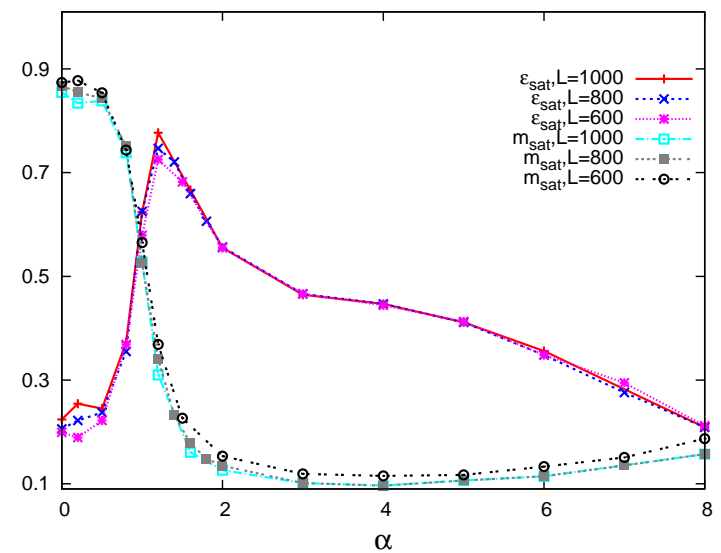

FIG. 8: (Color online) Saturation value of residual energy per spin $\varepsilon_{\text {sat }}$ and the saturation value of magnetisation $m_{\text {sat }}$ is plotted with $\alpha$ for Random Model B.

The value of $\alpha=\alpha_{\text {maxdis }}$, at which the maximum disorder occurs, decreases with the system size $L$ for small $L$ and then saturates for larger system sizes. The value of the residual energy at $\alpha_{\text {maxdis }}$ also increases with the system size (Fig. 9). This establishes the existence of the point of maximum disorder, for the RMB, at a finite value of $\alpha(\alpha \simeq 1.2)$ even in the thermodynamic limit. Similar to the RMA, here is a region beyond $\alpha=1.2$ where the energy and the magnetisation both decrease, until the magnetisation starts growing again. As already mentioned, this issue is addressed in sec. IV B.

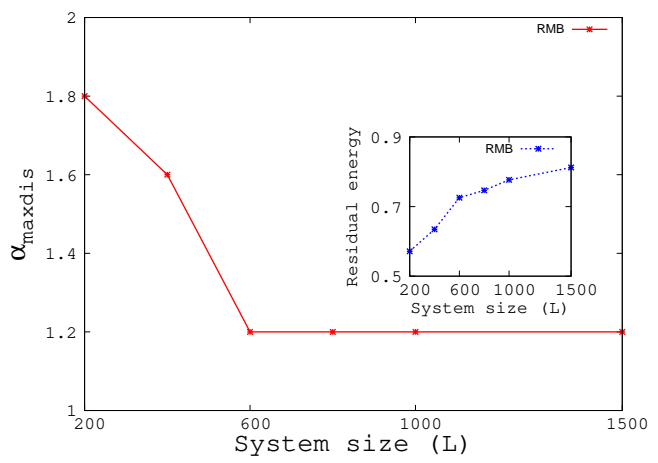

FIG. 9: (Color online) The value of $\alpha$ at the point of maximum disorder is plotted with the system size. Inset shows the increase of residual energy at the point of maximum disorder with the increment of the system size.

Saturation time for RMB in the random network in 
the region $0 \leq \alpha<1$ shows too large fluctuations to let one conclude whether it is a constant in this region or has a variation with $\alpha$. Beyond $\alpha=1$ and upto $\alpha=3.0$, it is almost independent of $\alpha$. For $\alpha>3$ the saturation time increases with $\alpha$. There is no remarkable finite size effect in the saturation time for the RMB for any finite value of $\alpha$. The saturation time varies as $L^{2}$ for a regular lattice corresponding to $\alpha \rightarrow \infty$, here it appears that for any finite $\alpha$, however large, there is no remarkable size dependence.

The freezing probability is small for $\alpha=0(\simeq 0.2)$ and increases rapidly with $\alpha$ for small $\alpha$. Freezing probability becomes almost unity beyond $\alpha \simeq 1.2$ and remains the same for large $\alpha$. It seems that for any finite $\alpha>1.2$ freezing probability remains unity and it will be zero only at $\alpha \rightarrow \infty$ (Fig. 10), as in one dimension, the freezing probability is zero for the regular network. So for RMB there is a discontinuity of freezing probability at $\alpha=\infty$ which corresponds to the $p=0$ point of RMA.

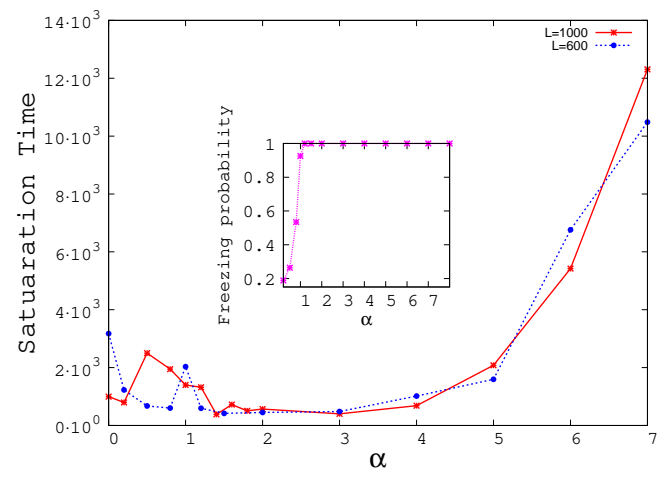

FIG. 10: (Color online) Time of saturation with the value of $\alpha$ is plotted for two different sizes for Random Model B. Inset shows the variation of freezing probability with $\alpha$ for RMB.

Beyond $\alpha \simeq 1.2$, the energy decreases with $\alpha$ though the freezing probability remains unity. This implies that although the system definitely reaches a frozen state, it approaches the real ground state monotonically as $\alpha \rightarrow$ $\infty$ (Fig. 8).

The above results indicate that, though for $\alpha>2$ the network behaves as a regular one, dynamically the network is regular only at its extreme value $\alpha \rightarrow \infty$.

We find that the persistence probability follows roughly a stretched exponential form with time (given by equation (2)) for any finite $\alpha$. The saturation value of the persistence, $P_{\text {sat }}$, does not depend on the system size. $P_{\text {sat }}$ changes with $\alpha$ and there exists an intermediate value of $\alpha$ where the value of $P_{\text {sat }}$ is maximum. For RMB also $b$ and $c$ vary nonmonotonically with $\alpha$ (Fig: 11).

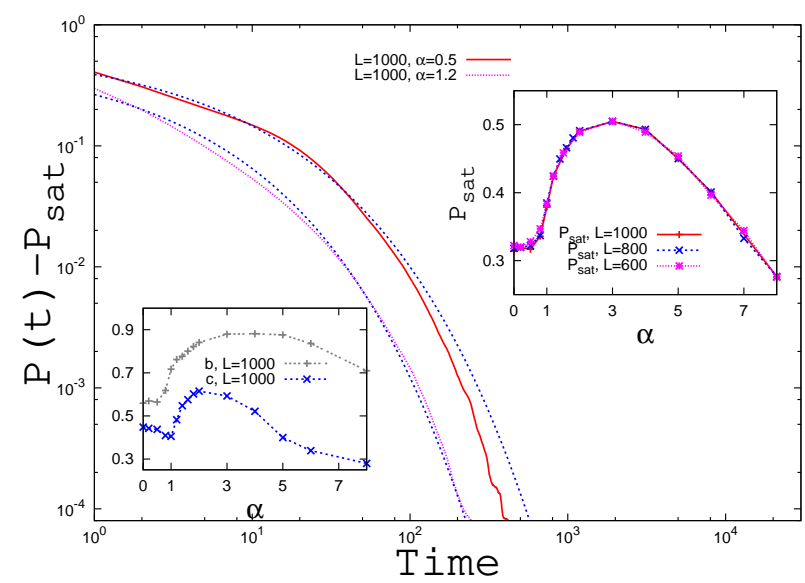

FIG. 11: (Color online) Decay of $P(t)-P_{\text {sat }}$ with time $t$ along with the stretched exponential function found to fit it approximately are shown. The inset in the top right shows the variation of the saturation value of persistence $P_{\text {sat }}$ with $\alpha$. The other inset on the bottom left shows the variation of $b$ and $c$ with $\alpha$.

\section{DISCUSSIONS ON THE RESULTS}

\section{A. Comparison of the results for RMA and RMB}

In the last two sections the results of a quench at zero temperature for the Ising model on RMA and RMB have been presented separately. In this subsection we will compare the results to understand how the difference in the nature of randomness affects the dynamics of Ising spin system.

The gross features of the results are similar: in both models we have a freezing effect which makes the system get stuck in a higher energy state compared to the static equilibrium state in which all spins are parallel. No power law scaling behaviour with time is observed in the dynamic quantities in either model. There exists a point in the parameter space where the deviation from the static ground state is maximum. The behaviour of the saturation times and freezing probability as functions of the disorder parameters are also quite similar qualitatively.

The saturation values of magnetisation and persistence attain a minimum and maximum value respectively at an intermediate value of the relevant parameters in both models. The decay of the persistence probability also follows the same functional form in the entire parameter space. The saturation values of the persistence has no size dependence for both the models. This indicates that as a whole the dynamics is not much affected due to the change in the nature of randomness of the Ising spin system.

Let us consider the parameter values at which the RMA and RMB are equivalent as a network: RMA and RMB behave as random networks at $p=1$ and $\alpha=0$ respectively. So one can expect that the saturation val- 
ues of residual energy per spin, magnetisation and the numerical value of the saturation time would be same at these values. However, the numerical values of these quantities are quite different. For RMA, at $p=1$ the saturation value of the residual energy per spin $\varepsilon_{\text {sat }} \simeq 0.415$ whereas for RMB at $\alpha=0 \varepsilon_{\text {sat }} \simeq 0.224$ for $L=1000$. Similarly we found numerically that for RMA the value of saturation magnetisation $m_{\text {sat }} \simeq 0.735$ for RMA and $m_{\text {sat }} \simeq 0.855$ for $\mathrm{RMB}$ for the same system size. This is because even though the networks are both random here, the connections have a subtle difference. For RMA, the number of second nearest neighbour is exactly zero at $p=1$ and all the other long range neighbour connections are equally probable. On the other hand, for RMB, second nearest neighbours can be still present in the network and the probability is same for this and any other longer range connection. This difference in the nature of randomness affects the dynamics of the Ising spin system sufficiently to make the saturation values different. This means that the systems are locked at different nonequilibrium steady states. For RMB, it is closer to the actual ground state as it is more short ranged in comparison.

The other values at which the two networks are equivalent are $p=0$ and $\alpha>2$ where regular network behaviour is found as far as the network properties are concerned. Interestingly, the behaviour of RMB even when $\alpha$ is finite and greater than 2 , is not quite like the dynamics of a regular one dimensional lattice with nearest and next nearest neighbour links only. In fact, the point at which the magnetisation becomes minimum is well inside the region $\alpha>2$ and not within the small world region as in RMA. Actually there is an extended region of regular and random network behaviour for the RMB, and as a result, a few more interesting points are possible to observe here. Only at the extreme point $\alpha=\infty$, the one dimensional Ising exponents $z=2.0$ and $\theta=0.375$ can be recovered as the frozen states continue to exist even for finite values of $\alpha>2$ for RMB. For the regular network with nearest and next nearest neighbour model, we have checked that there is no freezing at all. So discontinuities of the freezing probabilities occur at $p=0$ and $\alpha=\infty$ on RMA and RMA respectively.

Though the nature of randomness is different for RMA and RMB, for both the models there exists a point of maximum disorder where the saturation value of the residual energy per spin attains a maximum value. For $\mathrm{RMB}$, maximum disorder of the Ising spin system occurs near the static phase transition point (small world to random phase) whereas for RMA, the point of maximum disorder is well within the small world region.

We try to explain this considering the deviation from the point $p=1$ (for RMA) and $\alpha=0$ (for RMB). Two processes occur simultaneously here: (a) Number of connections with further neighbours decreases and (b) clustering becomes more probable. As a result of these two processes, freezing occurs. For RMA, the effect is less as there is less clustering [16]. But for RMB, the effect is more and spans the entire parameter space $\alpha>1$ and therefore the point of maximum disorder of Ising spin system is very close to the random - small world phase transition point $\alpha=1$.

The question may arise whether this difference prevails when the models are made even more similar. In RMB, the probability $p_{3}(\alpha)$ that $l \geq 3$ can be expressed as a function of $\alpha$ :

$$
p_{3}(\alpha)=\frac{\sum_{l=3}^{l=L / 2} l^{-\alpha}}{\sum_{l=2}^{l=L / 2} l^{-\alpha}} .
$$

A further correspondence between the two networks can be established by imposing $p=p_{3}(\alpha)$, which makes the number of second neighbour links in RMA and RMB also same (but the rest of the extra links are connected differently).

Using equation (3) we can obtain the value of $p$ corresponding to a given value of $\alpha$ and vice versa. But it is immediately seen that the two networks are not equivalent even after making them similar upto the second neighbour connections. For example, for $\alpha=2.0$, the corresponding value of $p=0.612$ in this scheme. But we have already seen that while the point of maximum disorder occurs close to this value of $p$ in RMA, the point of maximum disorder for RMB is considerably away from $\alpha=2.0$. So the nature of randomness continues to affect the dynamics at least quantitatively.

\section{B. Analysis of some general features of the quenching phenomena on networks}

We find several interesting features in the quenching phenomena of Ising spin systems on both the networks and in this subsection we attempt to provide an understanding of the same.

It is intriguing that the results indicate that the minimum amount of randomness can make the system freeze. What happens for small randomness? The interactions are still dominantly nearest neighbour type and domains in the conventional sense should grow which will be of both plus and minus signs. The system will freeze as there will be some stable domain walls due to the few long range interactions present. The domains, as the system attains saturation, will be small in number and large in size irrespective of their signs. As a result, the magnetisation attains a small value while the residual energy is still small.

This effect continues for some time till something more interesting happens. Take for example the case of quenching on RMA. There is a distinct region $0.4<p<$ 0.6 where the energy and magnetisation grow simultaneously, an apparently counterintuitive result. Similar behaviour can be noted for the quenching on RMB in a certain region in its parameter space. A problem to analyse the situation for different $p$ (or $\alpha$ ) values is that the final frozen states are not related in any way in principle. This is because the energy landscapes change as $p$ is changed 
and the initial configurations which undergo evolution are completely uncorrelated. In fact, in such a situation, even if the energy landscape is same with a number of local minima, different initial configurations will end up in different final nonequilibrium steady states. Nevertheless, one can attempt to explain this counterintuitive result assuming that the final states are not largely different when $p$ is changed slightly in the following way. This assumption and explanation are supported by the actual final states obtained for small system sizes.

Let us for example consider the RMA and take two values of $p, p_{2}>p_{1}$, and for which the magnetisation and residual energy of the final state corresponding $p_{2}$ are both larger than those for $p_{1}$. Now this can be possible due to the fragmentation of a larger domain into several domains such that the magnetisation increases. This can be demonstrated with a simple example: let us imagine a situation where one has only two domains of size $N^{+}$(of up spins) and $N^{-}$(of down spins) for $p_{1}$ with magnetisation equal to $m_{1}=\left|\left(N^{+}-N^{-}\right)\right| / L$ and assume that for $p_{2}$, the domain with $N^{+}$up spins remains same while the domain with $N^{-}$down spins gets fragmented into three domains of size $N^{-}{ }_{1}, N^{+}{ }_{1}$ and $N^{-}{ }_{2}$ in the final state. For $p_{2}$, therefore, the magnetisation is $m_{2}=\left|\left(N^{+}+2 N^{+}{ }_{1}-N^{-}\right)\right| / L$ which is larger than $m_{1}$. Here in this hypothetical case, we have assumed that $N^{+}>N^{-}$, and $p_{2}$ is very close to $p_{1}$. One can also assume that the energy increases for $p_{2}$ as the system is still sufficiently short ranged and the new domain walls cause an extra energy compared to the state obtained for $p_{1}$. Of course this is an oversimplified picture where we have assumed that the final states for $p_{1}$ and $p_{2}$ are identical except for the fragmentation of one domain. However, we find that the final configurations obtained for small systems for different values of $p$ as shown in Fig. 12 are consistent with our conjecture. These snapshots are representative of the real situation in the sense that they give a typical picture and are not just rare cases; we have obtained a similar picture from almost all such configurations generated for small systems.

As $p$ further increases, should the domains get fragmented into even smaller pieces? Answer is no, as the increasing number of long range interactions again help in the growth of so called domains, of one particular sign only such that the magnetisation grows and the energy decreases. However, domains of both signs still survive, although the sizes are no longer comparable. It can therefore be expected that the region for which both magnetisation and energy increase as a function of $p$ or $\alpha$ would continue till the short range interactions are dominating and our results are consistent with this expectation.

\section{SUMMARY AND CONCLUDING REMARKS}

In this paper, we addressed the question how the quenching dynamics of Ising spins depend on the nature

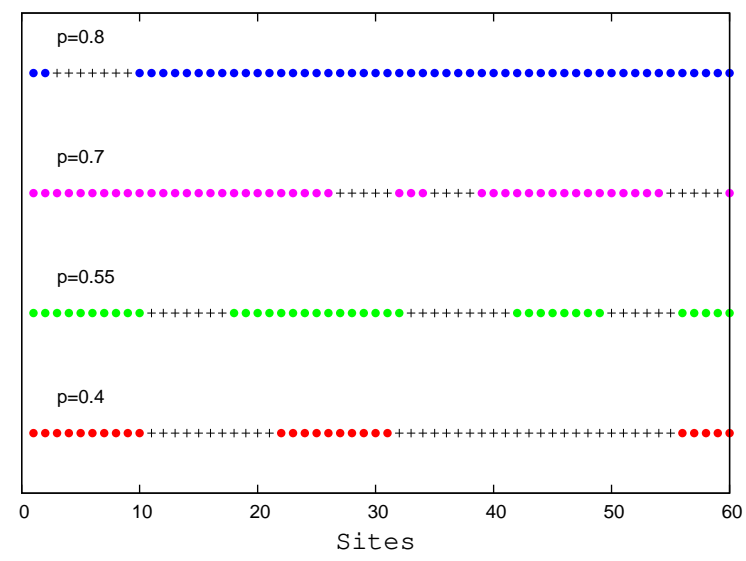

FIG. 12: (Color online) Snap shots of the final spin configurations for different values of the disorder parameter $p$ for quenching on RMA. The + and $\bullet$ signs indicate up and down spins respectively. The domains in the conventional sense are clearly visible.

of randomness of the underlying network by considering two networks in which the randomness is realised differently. The networks are same upto the first neighbour links and have same average degree per node. While the qualitative features are same, there are intricate differences occurring in the behaviour of the saturation values of the dynamical quantities.

Overall, we find some interesting features: the saturation values of the dynamical quantities do not have monotonic behaviour as a function of the disorder parameters. Especially, we find that increasing randomness does not necessarily make the system get locked in a higher energy state. The dynamics takes the system to a steady state very fast, and the saturation times are not dependent on the system size. No scaling behaviour is obtained from the studies either with time or with system size for any of the dynamic quantities. The most surprising result is perhaps the existence of a region in the parameter space where both the residual energy and the magnetisation increase which can be explained phenomenologically.

The Euclidean model, on which the study of the quenching of Ising spins is done for the first time to the best of our knowledge, shows some surprising behaviour both in the random and regular regions. We find that decreasing randomness makes the system end up in a higher energy state in the random region while in the regular region, familiar behaviour of the Ising dynamics with short range interactions are not obtained; in fact the probability of freezing is unity here indicating that in none of the realisation, the system could end up in the static ground state. The saturation time also does not show scaling with time.

As already mentioned, the present study is relevant for dynamical social phenomena on complex networks. For example, the evolution of binary opinions on a complex network (where the initial states are randomly +1 and -1 ) is analogous to the dynamical study reported in the 
present paper. Of course, in case of the opinion dynamics, the interactions could be more complex compared to the the simple Ising model. Our result indicates that the qualitative features of the results will not be much different for different complex networks.

Dynamic frustration [20] is responsible for freezing in many Ising systems where there is no frustration in the conventional sense. One interesting observation is that the nature of dynamic frustration in regular lattices of dimension greater than one and that in systems with random interaction (but no frustration) are in general quite different as in the latter one does not encounter the familiar scaling laws.

Acknowledgements :

Financial support from DST project SR/S2/CMP$56 / 2007$ and partial computational support from UPE project are acknowledged (SB and PS). SB acknowledges financial support from CSIR (Grant no. 09/028(0818)/2010-EMR-I).
[1] J. D. Gunton, M. San Miguel and P. S. Sahni, Phase Transitions and critical phenomena, Vol 8, eds. C. Domb and J. L. Lebowitz (Academic, NY 1983).

[2] A. J. Bray, Adv. Phys. 43357 (1994) and the references therein.

[3] B. Derrida, A. J. Bray and C. Godreche, J.Phys. A 27 L357 (1994).

[4] D. Stauffer, J. Phys. A 275029 (1994).

[5] P. L. Krapivsky, E. Ben-Naim and S. Redner, Phys. Rev. E 502474 (1994).

[6] V. Spirin, P. L. Krapivsky and S. Redner, Phys. Rev. E 63036118 (2001).

[7] C. K. Harris and R. B. Stinchcombe, Phys. Rev. Lett. 56, 869 (1986).

[8] S. Jain, J. Phys A 19 , L57 (1986); S. Jain, Phys. Rev. E 59, R2493 (1999).

[9] C. M. Newman, D. L. Stein, Phys. Rev. Lett. 82, 3944 3947 (1999).

[10] D. S. Fisher, P. L. Doussal and C. Monthus, Rhys Rev E 64, 066107 (2001).

[11] P. Svenson, Phys. Rev. E 64036122 (2001).

[12] O. Haggstrom, Physica A 310275 (2002).

[13] D. J. Watts and S. H. Strogatz, Nature 393, 440 (1998).

[14] D. Boyer and O. Miramontes, Phys. Rev. E 67, R035102 (2003).
[15] P. K. Das and P. Sen, Eur. Phys. J. B 47, 306 (2005).

[16] S. Goswami, S. Biswas and P. Sen Physica A 390, 972 (2011).

[17] J. Kleinberg, Nature 406, 845 (2000); S. Jespersen and A. Blumen, Phys. Rev. E 62, 6270 (2000). P. Sen and B. K. Chakrabarti, J. Phys A 34, 7749 (2001).

[18] P. Sen, K. Banerjee, T. Biswas, Phys. Rev. E 66037102 (2002); C. F. Moukarzel and M. A. de Menezes, Phys. Rev. E 65056709 (2002); P. Sen, K. Banerjee and T. Biswas, Phys. Rev. E 66, 037102 (2002); H. Zhu and ZX. Huang, Phys. Rev. E 70, 036117 (2004); A. Chatterjee and P. Sen, Phys. Rev. E 74036109 (2006); YuF. Chang, L. Sun and X. Cai, Phys. Rev. E 76021101 (2007); For a recent review on Euclidean networks, see P. Sen, in Recent Developments in Theoretical Physics, eds S. Ghosh and G. Kar, World Scientific, Singapore, 2010, p. 375.

[19] P. Sen and S. Dasgupta, J. Phys. A 3711949 (2004); P. Sen, Phys. Rev. E 81, 032103 (2010), S. Biswas and P. Sen, J. Phys. A 44145003 (2011).

[20] P. Sen and P. K. Das, in Quantum Annealing and other optimisation problems, eds. A. Das and B. K. Chakrabarti, Springer Verlag p 325 (2005). 